personalized nutrition strategies for tailoring gut microbes in the future. The study also complements other research $^{7-9}$ that explores how bacteria in the human gut might contribute to the body's response to a particular diet. Thanks to Patnode et al., we have fresh insights into how specific types of bacterium use and compete for dietary fibre. Future research will undoubtedly continue to refine the link between fibre-rich food and health, by taking into account the role of the gut microbial community.

Nathalie M. Delzenne and Laure B. Bindels are at the Louvain Drug Research Institute, Metabolism and Nutrition Research Group,

\title{
Infrared spectroscopy finally sees the light
}

\section{Andreas Barth}

The reliance of infrared spectroscopy on light transmission limits the sensitivity of many analytical applications. An approach that depends on the emission of infrared radiation from molecules promises to solve this problem. See p.52

Atoms in molecules oscillate when irradiated by infrared light. The particular light frequencies that drive these vibrations are absorbed by molecules, and depend on the molecules' chemical structure and environment. The infrared absorption spectrum of a sample can therefore be used as a molecular fingerprint by which to characterize its chemical composition. This has made infrared spectroscopy a widespread analytical technique. However, infrared spectra are difficult to measure for low concentrations of analytes and for samples in water. On page 52, Pupeza et al. ${ }^{1}$ present a concept for infrared spectroscopy that promises to alleviate these limitations.

Infrared light was discovered ${ }^{2}$ as a result of the problem it caused William Herschel while he was making astronomical observations of the Sun - it created a disturbing heating sensation in his eye that he wanted to filter out. Today, however, the benefits of infrared radiation for a multitude of analytical purposes are widely appreciated. Its applications range from the detection of molecules in outer space $^{3,4}$, including that of water on Mars ${ }^{5}$, to deciphering the molecular mechanisms of proteins in living organisms $s^{6,7}$. In the everyday world, it is used in food analysis ${ }^{6,8}$ and in forensic police investigations $s^{6,9}$, for example. Much research is being done to bring infrared spectroscopy to the clinic, because the
Catholic University of Louvain, 1200 Brussels, Belgium.

e-mail: nathalie.delzenne@uclouvain.be

1. Patnode, M. L. et al. Cell 179, 59-73 (2019). j.clnu.2019.03.002 (2019).

3. Bindels, L. B., Delzenne, N. M., Cani, P. D. \& Walter, J. Nature Rev. Gastroenterol. Hepatol. 12, 303-310 (2015).

4. Gibson, G. R. et al. Nature Rev. Gastroenterol. Hepatol. 14, 491-502 (2017).

5. Ridaura, V. K. et al. Science 341, 1241214 (2013)

6. The Human Microbiome Project Consortium. Nature 486, 207-214 (2012).

7. Salonen, A. et al. ISME J. 8, 2218-2230 (2014).

9. Zhao, L. et al. Science 359, 1151-1156 (2018).

This article was published online on 4 December 2019.
2. Delzenne, N. M. et al. Clin. Nutr. https://doi.org/10.1016/

8. Bindels, L. B. et al. Microbiome 5, 12 (2017) analysis of biological tissue and body fluids can be used to detect and diagnose disease $e^{6,7,10}$.

One of the main obstacles to the infrared analysis of biological samples is the strong absorption of infrared radiation by water - a problem that limits the sample thickness to less than 10 micrometres for most purposes. This issue also makes it difficult to add aqueous solutions of reagents (such as acids or salts) to samples to manipulate the state of molecules in the sample. Such manipulations are desirable, for example, for studying the binding of small molecules to proteins, and are standard practice when using ultraviolet or visible spectroscopy. Furthermore, because infrared radiation is absorbed by water, samples must often be concentrated or dried.

Pupeza and colleagues report a solution to this problem. They irradiate samples with an ultrashort pulse (on the scale of femtoseconds; $1 \mathrm{fs}$ is $10^{-15}$ seconds) of mid-infrared light. Specific frequencies of the light are absorbed by sample molecules, generating vibrations. These vibrations continue after the pulse has ended, and last until the vibrational energy is dissipated to the environment (which takes a few picoseconds; $1 \mathrm{ps}$ is $10^{-12} \mathrm{~s}$ ). Because the vibrating atoms carry partial electrical charges, their oscillations generate electromagnetic radiation, similar to the way in which oscillating electrons produce electromagnetic radiation in an antenna. The generated radiation has the same frequency as that of the molecular vibrations, and so carries information about all of the sample molecules - the authors therefore call it a global molecular fingerprint. It is measured using a second ultrashort pulse of light, this time in the near-infrared spectral range, through a method called electro-optic sampling ${ }^{11}$.

The authors' approach is conceptually different from conventional absorption

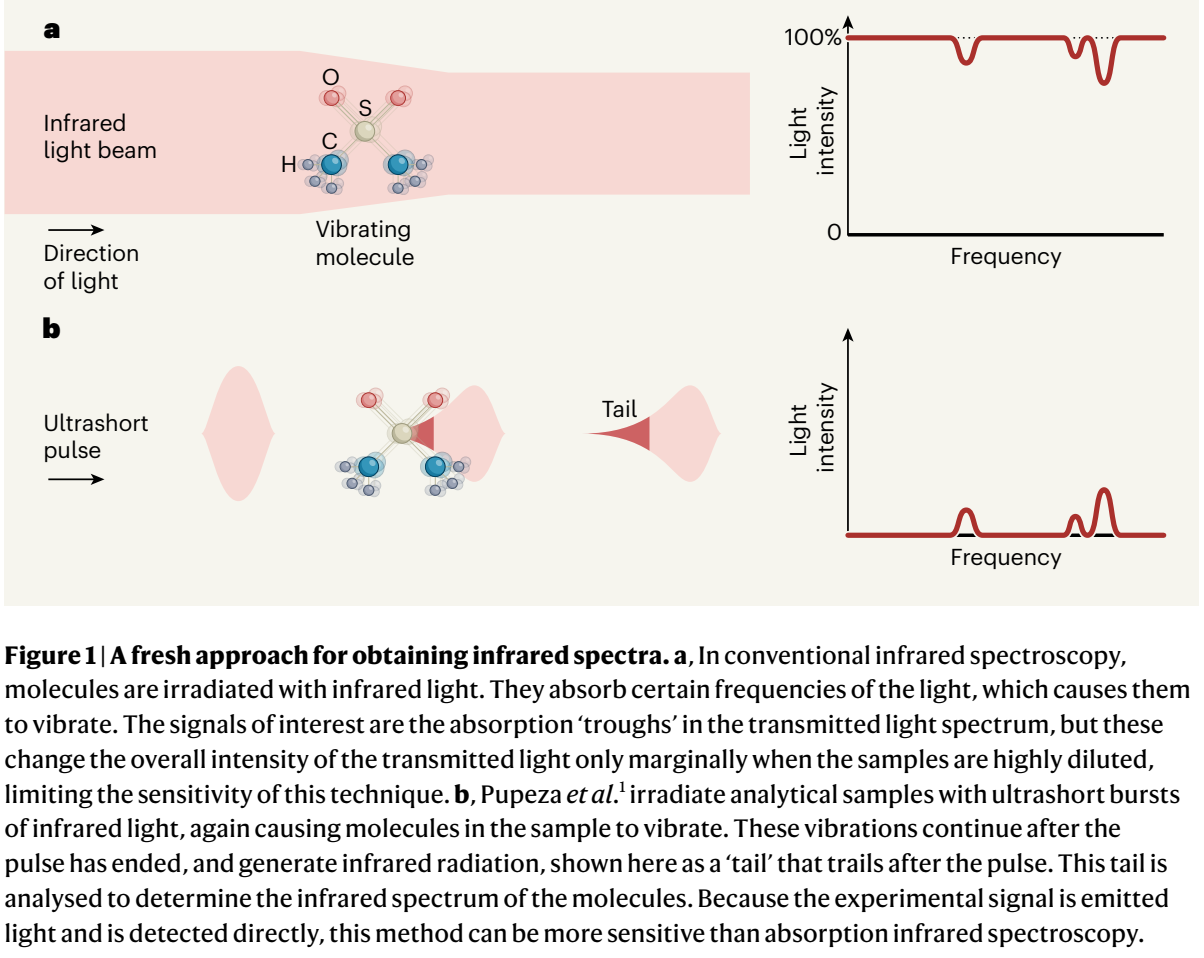


measurements. In absorption spectroscopy, the signal is sensed only indirectly, from the light that does not interact with the sample (Fig. 1a). Weak absorption is therefore very difficult to detect, because it changes the intensity of the transmitted light only marginally. Theoretically, the detection of weak absorbers could be improved by increasing the intensity of the incident light, but commonly used infrared detectors become less sensitive at higher light intensities ${ }^{12}$, imposing a practical limit on the maximum light intensity that can be used. By contrast, Pupeza et al. detect the signal of interest - the radiation emitted from the vibrating molecules - directly (Fig. 1b). This is analogous to the difference between absorbance and fluorescence measurements in the visible spectral range: fluorescence measurements are the more sensitive because they detect a signal directly from the sample, and can even detect it from a single molecule.

Pupeza and colleagues demonstrate the high sensitivity of their approach in various ways. For example, they were able to detect 40 -fold lower concentrations of a compound in solution, and to better distinguish between two similar compounds, than when using absorption spectroscopy. They also obtained spectra of biological samples that block nearly all of the incoming light (in one case, at least 99.999\%). Thus, the new approach senses light where currently used methods see only darkness. This is an impressive achievement, and might alleviate both of the main problems of conventional infrared spectroscopy: sensitivity and strong infrared absorption by water. It will simplify sample preparation in many cases by removing the need for sample concentration or drying, and will open up new applications - particularly those involving aqueous biological samples.

The authors suggest several ideas for taking the method further, such as by increasing the power of the laser used to irradiate the sample. It is to be hoped that such measures will further narrow the technological gap that at present prevents the method from achieving the ultimate goal of single-molecule sensitivity in bulk water. Other challenges will be to increase the spectral range of the measurements to include the shorter wavelengths at which prominent and diagnostically useful signals are found for proteins, lipids and nucleotides, and to develop a spectrometer suitable for commercialization at a competitive price.

Andreas Barth is in the Department of Biochemistry and Biophysics, Stockholm University, Stockholm 106 91, Sweden. e-mail: barth@dbb.su.se

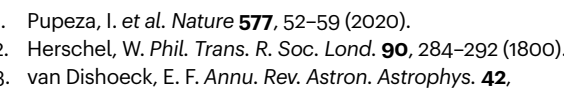

4. Ehrenfreund, P. \& Charnley, S. B. Annu. Rev. Astron Astrophys. 38, 427-483 (2000)

5. Bibring, J.-P. et al. Nature 428, 627-630 (2004)

6. Chalmers, J. M. \& Griffiths, P. R. (eds) Handbook of Vibrational Spectroscopy Vols 4 \& 5 (Wiley, 2001).

7. Barth, A. \& Haris, P. I. (eds) Biological and Biomedical Infrared Spectroscopy (IOS, 2009).

8. Sun, D.-W. (ed.) Infrared Spectroscopy for Food Quality Analysis and Control (Academic, 2008)
9. Chalmers, J. M., Edwards, H. G. M. \& Hargreaves, M. D. (eds) Infrared and Raman Spectroscopy in Forensic Science (Wiley, 2012).

10. Bunaciu, A. A., Fleschin, S., Hoang, V. D. \& Aboul-Enein, H. Y. Crit. Rev. Anal. Chem. 47, 67-75 (2017).

11. Wu, Q. \& Zhang, X.-C. Appl. Phys. Lett. 67, 3523-3525 (1995)

12. Theocharous, E., Ishii, J. \& Fox, P. N. Appl. Opt. 43. 4182-4188 (2004)

Medical research

Etta D. Pisano

Could artificial intelligence improve the accuracy of

screening for breast cancer? A comparison of the diagnostic

performance of expert physicians and computers suggests so,

but the clinical implications are as yet uncertain. See p.89

Screening is used to detect breast cancer early in women who have no obvious signs of the disease. This image-analysis task is challenging because cancer is often hidden or masked in mammograms by overlapping 'dense' breast tissue. The problem has stimulated efforts to develop computer-based artificial-intelligence (AI) systems to improve diagnostic performance. On page 89 , McKinney et al. ${ }^{1}$ report the development of an AI system that outperforms expert radiologists in accurately interpreting mammograms from screening programmes. The work is part of a wave of studies investigating the use of $\mathrm{Al}$ in a range of medical-imaging contexts ${ }^{2}$.

Despite some limitations, McKinney and colleagues' study is impressive. Its strengths include the large scale of the data sets used for training and subsequently validating the $\mathrm{AI}$ algorithm. Mammograms for 25,856 women in the United Kingdom and 3,097 women in the United States were used to train the AI system. The system was then used to identify the presence of breast cancer in mammograms of women who were known to have had either biopsy-proven breast cancer or normal follow-up imaging results at least 365 days later. These outcomes are the widely accepted gold standard for confirming breast cancer status in people undergoing screening for the disease. The authors report that the Al system outperformed both the historical decisions made by the radiologists who initially assessed the mammograms, and the decisions of 6 expert radiologists who interpreted 500 randomly selected cases in a controlled study.

McKinney and colleagues' results suggest that $\mathrm{AI}$ might some day have a role in aiding the early detection of breast cancer, but the authors rightly note that clinical trials will be needed to further assess the utility of this tool in medical practice. The real world is more complicated and potentially more diverse than the type of controlled research environment reported in this study. For example, the study did not include all the different mammography technologies currently in use, and most images were obtained using a mammography system from a single manufacturer. The study included examples of two types of mammogram: tomosynthesis (also known as 3D mammography) and conventional digital (2D) mammography. It would be useful to know how the system performed individually for each technology.

\section{"Clinical trials will be needed to further assess the utility of this tool in medical practice."}

The demographics of the population studied by the authors is not well defined, apart from by age. The performance of $\mathrm{AI}$ algorithms can be highly dependent on the population used in the training sets. It is therefore important that a representative sample of the general population be used in the development of this technology, to ensure that the results are broadly applicable.

Another reason to temper excitement about this and similar AI studies is the lessons learnt from computer-aided detection (CAD) of breast cancer. CAD, an earlier computer system aimed at improving mammography interpretation in the clinic, showed great promise in experimental testing, but fell short in real-world settings ${ }^{3}$. CAD marks 